\title{
Bundesgesetzgebungskompetenz für die Bedarfsfeststellung eines einzelnen Braunkohletagebaus?*
}

\author{
Georg Hermes
}

() Der/die Autor(en) 2021. Dieser Artikel ist eine Open-Access-Publikation.

\begin{abstract}
Im Gesetzgebungsverfahren zur Umsetzung des Kohleausstiegs wurde kurz vor den abschließenden Beratungen auf Initiative des Ausschusses für Wirtschaft und Energie eine Vorschrift in das Gesetz zur Reduzierung und zur Beendigung der Kohleverstromung ${ }^{1}$ (KVBG) aufgenommen, die die „Notwendigkeit" des Braunkohletagebaus Garzweiler II rechtlich absichern soll (S48 KVBG; dazu 1). Unabhängig von der Frage, ob dieser Versuch durch die verschärften Jahresemissionsmengen für die Energiewirtschaft, ${ }^{2}$ die durch die Entscheidung des Bundesverfassungsgerichts vom 24. 3. $2021^{3}$ veranlasst sind, bereits als überholt angesehen werden kann, und auch unabhängig von materiellen Bedenken ${ }^{4}$ fehlt dem Bund die Gesetzgebungskompetenz. Denn die Vorschrift ist an dem Erforderlichkeitsmaßstab des Art. 72 Abs. 2 GG zu messen (dazu 2) und die Anwendung der dazu in der Rechtsprechung entwickelten Kriterien (dazu 3) auf $\$ 48$ KVBG führt zu dem Ergebnis, dass es an der Erforderlichkeit der Bedarfsfeststellung für Garzweiler II zur Wahrung der Wirtschaftseinheit im gesamtstaatlichen Interesse fehlt (dazu 4).
\end{abstract}

\section{Die Regelung zum Tagebau Garzweiler II im} Kontext der Beendigung der Braunkohleverstromung

Das Gesetz zur Reduzierung und zur Beendigung der Kohleverstromung und zur Anderung weiterer Gesetze (Kohleausstiegsgesetz) vom 8. August 2020 (BGB1. Nr. 37, S. 1818) enthält als Artikel 1 das Gesetz zur Reduzierung und zur Beendigung der Kohleverstromung (Kohleverstromungsbeendigungsgesetz - KVBG), das die schrittweise und möglichst stetige Reduzierung und Beendigung der Erzeugung elektrischer Energie durch den Einsatz von Kohle in Deutschland regelt ( $\$ 1$ Abs. 1 Satz 2 KVBG). Die in $\$ 2$ Abs. $2 \mathrm{KVBG}$ enthaltenen und in $\$ 4 \mathrm{KVBG}$ näher konkretisierten Reduktionsziele sind bezogen auf die verbleibende elektrische Nettonennleistung von Anlagen am Strommarkt zur Erzeugung elektrischer Energie durch den Einsatz von Kohle in Deutschland, die im Kalenderjahr 2022 auf 15 Gigawatt Steinkohle und 15 Gigawatt Braunkohle, im Kalenderjahr 2030 auf 8 Gigawatt Steinkohle und 9 Gigawatt Braunkohle und spätestens bis zum Ablauf des Kalenderjahres 2038 auf 0 Gigawatt Steinkohle und 0 Gigawatt Braunkohle schrittweise und stetig reduziert werden sollen.

$1.1 \int 48$ KVBG als Element der Regelungen zur Reduzierung der Braunkohleverstromung

Während für die Anlagen zur Erzeugung elektrischer Energie aus Steinkohle die Reduzierung durch eine Kombination aus Ausschreibungen ( $\$ S 10 \mathrm{ff}$. KVBG) und gesetzlicher Reduzierung mit behördlichen Anordnungen ( $\$ S 27 \mathrm{ff}$. KVBG) bewirkt wird, sieht das Gesetz für die Reduzierung der Braunkohleverstromung (Teil 5, \$\$ $40 \mathrm{ff}$. KVBG) feste Stilllegungsdaten für die einzelnen Braunkohleanlagen vor ( 440 Abs. 1 i.V.m. Anlage 2 KVBG), die zwischen dem 31.12.2020 (Niederaußem D) und dem 31.12.2038 (Nie-

Prof. Dr. Georg Hermes,

Professur für offentliches Recht,

Goethe-Universität Frankfurt am Main,

Frankfurt am Main, Deutschland deraußem, Neurath, Schwarz Pumpe, Boxberg) liegen. Die Betreiber von Braunkohlekraftwerken können ihre Anlagen auch bereits vor diesen Zeitpunkten stilllegen, wobei allerdings der Versorgungssicherheit dienende Regeln über die Netzreserve (\$42 KVBG i. V.m. \$\$ 13 b und 13c Energiewirtschaftsgesetz [EnWG]) gelten.

Bestandteil des Teils 5 des Kohleverstromungsbeendigungsgesetzes ist auch die hier interessierende Vorschrift des $\$ 48 \mathrm{KVBG}$, die folgenden Wortlaut hat:
, $\int 48$
Energiepolitische und energiewirtschaftliche Notwendigkeit des Tagebaus Garzweiler II
(1) Die energiepolitische und energiewirtschaftliche Notwen- digkeit und der vordringliche Bedarf zur Gewährleistung ei- ner sicheren und zuverlässigen Energieversorgung wird für den Tagebau Garzweiler II in den Grenzen der Leitentscheidung der Landesregierung von Nordrhein-Westfalen zur Zukunft des Rheinischen Braunkohlereviers/Garzweiler II vom 5. Juli 2016 festgestellt.
(2) Die Feststellung nach Absatz 1 ist für die Planung sowie fachrechtliche Zulassungen zu Grunde zu legen. Der damit verbindlich festgestellte energiepolitische und energiewirtschaft- liche Bedarf schließt räumliche Konkretisierungen im Rahmen der Braunkohlenplanung und der anschließenden fachrecht- lichen Zulassungen des Landes Nordrhein-Westfalen nicht aus."

\subsection{Der Regierungsentwurf (öffentlich-rechtlicher Vertrag)}

Im Gesetzentwurf der Bundesregierung (BT-Drs. 19/17342) war diese Vorschrift noch nicht vorgesehen. Allerdings enthielt die im Entwurf der Bundesregierung vorgesehene Ermächtigung der Bundesregierung zum Abschluss eines öffentlich-rechtlichen Vertrages mit den Betreibern von Braunkohleanlagen und weiteren, von der Reduzierung und Beendigung der Braunkohleverstromung unmittelbar betroffenen Braunkohletagebauunternehmen ( $\$ 42$ des Entwurfs) im Rahmen einer detaillierten Auflistung der Gegenstände des Vertrages auch den folgenden Vertragsgegenstand:

„7. die Feststellung der energiewirtschaftlichen Notwendigkeit des Tagebaus Garzweiler inklusive des 3. Umsiedlungsabschnitts in den Grenzen der Leitentscheidung der Landesregierung von Nordrhein-Westfalen zur Zukunft des Rheinischen Braunkohlereviers/Garzweiler II vom 5. Juli 2016". Für den Fall des Scheiterns eines öffentlich-rechtlichen Vertrages sah $\int 43$ des Regierungsentwurfs eine Verordnungsermächtigung

^) Der Beitrag beruht auf einem Kurzgutachten im Auftrag der Bundestagsfraktion Bündnis 90/Die Grünen, das der Verf. im Februar 2021 erstattet hat.

1) V. 8.8.2020, BGB1. I S. 1818; zuletzt geändert durch Art. 26 Abs. 2 des Finanzmarktintegritätsstärkungsgesetzes v. 3.6.2021, BGB1. I S. 1534

2) S. den Entwurf der Bundesregierung eines Ersten Gesetzes zur Änderung des Bundes-Klimaschutzgesetzes, BR-Drs. 411/21 (Anlagen 2 und $3 \mathrm{zu} \$ 4$ ).

3) BVerfG, Beschl. v. 24.3.2021 - 1 BvR 2656/18

4) S. dazu Frenz, ZNER 2020, S. 492 ff. 
für denselben Gegenstand vor ( $\int 43$ Abs. 2 Nr. 6 des Regierungsentwurfs).

Auf eine Kleine Anfrage zu dieser Regelung im Gesetzentwurf der Bundesregierung antwortete diese wie folgt (BTDrs. 19/18987, S. 3):

\begin{abstract}
„Die Bundesregierung setzt mit der Feststellung der energiewirtschaftlichen Notwendigkeit des Tagebaus Garzweiler den Kompromiss aus der Bund-Länder-Vereinbarung zum Kohleausstieg vom 15. Januar 2020 um. Die Leitentscheidung des Landes Nordrhein-Westfalen aus dem Jahr 2016 und der Kompromiss aus der Bund-Länder-Vereinbarung zum Kohleausstieg vom 15. Januar 2020 widersprechen sich nicht. Vielmehr wird der Tagebau Garzweiler in den Grenzen der Leitentscheidung inklusive des 3. Umsiedlungsabschnitts in der Bund-Länder-Vereinbarung bestätigt. Ein Unterschied zur damaligen Leitentscheidung ergibt sich lediglich für den Tagebau Hambach: So sah die Leitentscheidung noch die vollständige Auskohlung des Tagebaus vor. Die Bund-LänderEinigung sieht hingegen vor, dass der Hambacher Forst gemäß Empfehlung der Kommission „Wachstum, Strukturwandel und Beschäftigung" nicht für den Tagebau in Anspruch genommen wird. Da sich die Feststellung der energiewirtschaftlichen Notwendigkeit des Tagebaus Garzweiler in den Grenzen der Leitentscheidung der Landesregierung von NordrheinWestfalen zur Zukunft des Rheinischen Braunkohlereviers/ Garzweiler II vom 5. Juli 2016 bewegt, wird die Wirkung dieser Leitentscheidung insoweit nicht beeinträchtigt."
\end{abstract}

\subsection{Bund-/Ländereinigung vom 15. Januar 2020}

Bei der in dieser Antwort der Bundesregierung in Bezug genommenen ,Bund-Länder-Vereinbarung zum Kohleausstieg vom 15. Januar 2020" handelt es sich nach der Pressemitteilung 21/20 des Presse- und Informationsamtes der Bundesregierung vom 16. Januar $2020^{5}$ um eine „Besprechung der Bundeskanzlerin mit BM Scholz, BM Altmaier, BM'in Schulze, BM Braun sowie MP Woidke (BB), MP Laschet (NW), MP Kretschmer (SN) und MP Haseloff (ST) am 15.1.2020“, in deren Rahmen die Bundesregierung ,den Ministerpräsidenten der Länder Brandenburg, Nordrhein-Westfalen, Sachsen und Sachsen-Anhalt einen Stilllegungspfad für die Braunkohlekraftwerke in Deutschland vorgestellt [hat], den sie beabsichtigt mit den Betreibern der Braunkohle-Kraftwerke und -Tagebaue vertraglich festzulegen“. Die Ministerpräsidenten haben diesem Stilllegungspfad zugestimmt. Weiter heißt es in der Pressemitteilung zu den Ergebnissen der Besprechung:

\section{„4. Zum Zwecke der Energieversorgungssicherheit wird die energiewirtschaftliche Notwendigkeit des Tagebaus Garzwei- ler in den Grenzen der Leitentscheidung aus dem Jahr 2016 inklusive des 3. Umsiedlungsabschnitts im Kohleausstiegsge- setz festgestellt."}

\subsection{Die Ausschussbegründung zu $\int 48$ KVBG}

\48 KVBG wurde eingefügt durch die Beschlussempfehlung des Ausschusses für Wirtschaft und Energie, ${ }^{6}$ die u.a. den Teil zur Reduzierung und Beendigung der Braunkohleverstromung erheblich veränderte und insbesondere $\mathrm{Ge}-$ genstände, die nach dem Regierungsentwurf Bestandteil des öffentlich-rechtlichen Vertrages werden sollten, unmittelbar im Gesetz selbst einer Regelung zuführte. Die Begründung lautet wie folgt:

„In $\int 48$ wird die energiepolitische und energiewirtschaftliche Notwendigkeit des Tagebaus Garzweiler II in dem dort benannten Umfang festgestellt. Diese Feststellung ist für landesrechtliche Planungs-und Genehmigungsverfahren verbindlich. Übereinstimmend mit den Ergebnissen der Kommission
„Wachstum, Strukturwandel und Beschäftigung" (KWSB) sowie den Ergebnissen der Einigung zwischen Bund, Ländern und den Betreibern der Braunkohlekraftwerke vom 15. Januar 2020, besteht der Bedarf für eine zeitnahe und planungssichere Umsetzung des Konsenses zur Stilllegung von Braunkohlekraftwerken und den im Verbund betriebenen Braunkohletagebauen im Rahmen der einschlägigen Planungs-und Genehmigungsverfahren. Dies betrifft die Umplanung (u. a. flächige Ausdehnung, Wiedernutzbarmachung, Restseegestaltung) bestehender Tagebaue im Hinblick auf einen angepassten Kohlebedarf. Dieser muss sowohl mit dem sich verkleinernden Kraftwerkspark und den in Anlage 2 zu diesem Gesetz festgelegten Stillegungsdaten im Einklang stehen, als auch die sichere und zuverlässige Energieversorgung auf dem Weg bis zum vollständigen Ausstieg aus der Kohleverstromung im Sinne der staatlichen Verantwortung für die Energieversorgung als Aufgabe der Daseinsvorsorge gewährleisten. Der Bund hat für die Umsetzung des Gesamtkompromisses zum Ausstieg aus der Kohleverstromung ein weites gesetzgeberisches Ermessen. Die politische Entscheidung für den Kohleausstieg beinhaltet u.a. mit Unsicherheiten behaftete Prognosen sowie grundlegende Abwägungsentscheidungen über den künftigen Energiebedarf, die Deckung dieses Energiebedarfs durch unterschiedliche Technologien, die dafür erforderliche Rohstoffversorgung in Deutschland sowie die wirtschaftlichen und sozialen Folgen der Kraftwerks-und Tagebaustilllegungen. Der Ausstieg aus der Kohleverstromung ist eine normative Grundsatzentscheidung im Bereich der Energieversorgung.

Für solche Grundsatzentscheidungen hat der Gesetzgeber einen besonders großen Gestaltungsspielraum, wie das Bundesverfassungsgericht in seinem Urteil über den Atomausstieg anerkannt hat:

„Im Hinblick auf diese Besonderheiten der Kernenergienutzung hat das Bundesverfassungsgericht bereits in seiner Kalkar-Entscheidung betont, dass die normative Grundsatzentscheidung für oder gegen die rechtliche Zulässigkeit der friedlichen Nutzung der Kernenergie dem parlamentarischen Gesetzgeber vorbehalten ist (BVerfGE 49, 89, 127) und dass dem Atomrecht eine Sonderstellung zukommt, die es rechtfertigt, von verfassungsrechtlichen Grundsätzen abzuweichen, die auf anderen Rechtsgebieten anerkannt sind (a. a. O. S. 146). Daraus folgt ein großer Gestaltungsspielraum des Gesetzgebers bei der Entscheidung über das Ob und Wie der friedlichen Nutzung der Kernenergie. Eine völlige Freistellung von ansonsten gebotenen Ausgleichsregelungen ist damit jedoch nicht verbunden." (BVerfG, Urteil v. 6. 12.2016-1 BvR 2821/11, BVerfGE 143, 246-396, juris, Rdnr. 298). Diese Erwägungen des Bundesverfassungsgerichts zum Atomausstieg sind auch auf die Entscheidung zum Ausstieg aus der Kohleverstromung und die Entscheidung zur in diesem Zusammenhang verbleibenden Restnutzung von einzelnen Braunkohlekraftwerken sowie den dafür erforderlichen Braunkohletagebauen übertragbar. Daher wird im Rahmen des beschriebenen Gestaltungsspielraums des Gesetzgebers die energiepolitische bzw. energiewirtschaftlichen Notwendigkeit des Tagebaus Garzweiler II in den Grenzen der Leitentscheidung der Landesregierung von Nordrhein-Westfalen zur Zukunft des Rheinischen Braunkohlereviers/Garzweiler II vom 5. 7. 2016 festgestellt. Bei dieser Feststellung handelt es sich um eine Form der gesetzlichen Bedarfsplanung in dem politisch besonders diskutierten Bereich der Braunkohlennutzung. Die Regelung fügt sich ein in einen politischen Kompromiss zum Kohleausstieg. In diesem Kompromiss zu einem

5) Dort finden sich die folgenden Zitate, Stand 13.6.2021, abrufbar unter https://www.bmwi.de/Redaktion/DE/Downloads/B/ bund-laender-einigung-zum-kohleausstieg.pdf?__blob=publicationFile \& $\mathrm{v}=8$.

6) BT-Drs. 19/20714 (neu), S. 73. 
Kohleausstiegsgesetz haben sich Bund und Länder auf ein Verfahren zum Kohleausstieg geeinigt. Ziele sind die Einhaltung der internationalen, europäischen und nationalen Klimaziele bei gleichzeitiger Sicherung der Energieversorgung in Deutschland und einer langfristig vorhersehbaren sowie belastbaren Planung für die betroffenen Braunkohleregionen und die dort beschäftigten Arbeitnehmerinnen und Arbeitnehmer. Teil dieser normativen Grundsatzentscheidung ist es, dass in einem bestimmten Umfang in einem bestimmten Tagebau weiterhin die Rohstoff-und Energieversorgung durch einen bereits begonnenen und langfristig geplant und genehmigten Braunkohlentagebau gesichert werden soll. Es handelt sich um einen politisch sensiblen Bereich mit langfristiger Planung. Die Versorgung der Braunkohlekraftwerke muss planbar und belastbar gesichert werden. Im Rahmen des Gesamtkompromiss zum Kohleausstieg wurde vereinbart, dass der Hambacher Forst gemäß Empfehlung der KWSB entgegen der bisherigen Genehmigung nicht für den Tagebau in Anspruch genommen wird. Damit verbleibt Garzweiler II als einziger Tagebau zur planungssicheren Versorgung der Braunkohlekraftwerke in Neurath und Niederaußem, woraus sich unter Beachtung aller Prognoseunsicherheiten ebenfalls die energiepolitische bzw. energiewirtschaftliche Notwendigkeit des Tagebaus Garzweiler II in den Grenzen der Leitentscheidung der Landesregierung von Nordrhein-Westfalen ergibt. Indem sich der Gesetzgeber hier vorhandene Bewertungen und Planungen in Teilen zu Eigen machen möchte, stellt er eine eigene Abwägungsentscheidung an. Ergänzend ist darauf hinzuweisen, dass diese bundesgesetzliche Feststellung der energiewirtschaftlichen Notwendigkeit des Tagebaus Garzweiler II die besondere Situation im Rheinischen Revier und insbesondere beim Tagebau Garzweiler II adressiert. Dies bedeutet nicht, dass andere Tagebaue nicht energiewirtschaftlich und energiepolitische erforderlich sind. Insofern hat diese Feststellung keine nachteiligen Auswirkungen auf die Planungs- und Genehmigungsverfahren anderer Tagebaue."

\subsection{Verfassungsrechtliche Zweifel}

Bei der Regelung des $₫ 48$ KVBG handelt es sich offensichtlich um eine isolierte, mit einer Prüfung der energiepolitischen und energiewirtschaftlichen Notwendigkeit anderer Braunkohletagebaue in Deutschland nicht in Zusammenhang stehende Einzelfallregelung. Daraus resultieren Zweifel an der Kompetenz des Bundes, eine solche gesetzliche Regelung zu treffen. Diese Zweifel stützen sich insbesondere darauf, dass es an der in Art. 72 Abs. 2 GG vorausgesetzten Erforderlichkeit zur Wahrung der Wirtschaftseinheit im gesamtstaatlichen Interesse fehlen könnte. Deshalb wird nachfolgende zunächst begründet, dass $₫ 48 \mathrm{KVBG}$ den Anforderungen des Art. 72 Abs. 2 GG unterliegt (dazu 2), bevor die Anforderungen an die Erforderlichkeit eines Gesetzes zur Wahrung der Wirtschaftseinheit im gesamtstaatlichen Interesse genauer in den Blick genommen werden (3). Die Prüfung des $\ 48$ KVBG an diesen Anforderungen führt zu einem negativen Ergebnis (dazu 4).

\section{Unterliegt $\$ 48 \mathrm{KVBG}$ den Anforderungen des Art. 72 Abs. 2 GG?}

Damit Art. 72 Abs. 2 GG hier zur Anwendung kommen kann, muss es sich bei $\$ 48$ KVBG um eine Regelung auf einem der in Art. 72 Abs. 2 GG explizit genannten Gebiete handeln. Hier kommt nur eine Regelung auf dem Gebiet des Rechts der Wirtschaft nach Art. 74 Abs. 1 Nr. 11 GG in Betracht, wobei von den in Klammern benannten speziellen Wirtschaftssektoren für $\$ 48$ KVBG sowohl der „Bergbau" ${ }^{8}$ als auch die ,Energiewirtschaft" in Betracht kommen. Bei der Braunkohle handelt es sich gem. \$3 Abs. 2 BBergG um einen bergfreien Bodenschatz, dessen Gewinnung sich - auf der Grundlage landesplanungsrechtlicher Vorbereitung $^{9}$ - nach dem Bundesberggesetz (BBergG $)^{10}$ richtet, das kompetenzrechtlich auf Art. 74 Abs. 1 Nr. 11 GG (Bergbau) gestützt ist. Da $\$ 48$ KVBG den Braunkohletagebau Garzweiler II zum Gegenstand hat, könnte dies für den Gesetzgebungskompetenztitel „Bergbau“ sprechen. Allerdings liegt bereits nach dem Wortlaut der maßgebliche Regelungsgehalt des $\$ 48$ KVBG darin, dass die energiepolitische und energiewirtschaftliche Notwendigkeit und der vordringliche Bedarf zur Gewährleistung einer sicheren und zuverlässigen Energieversorgung für den Tagebau Garzweiler II festgestellt wird. Es geht der Regelung also nicht um die bergrechtsspezifischen Belange der Gewinnung des Bodenschatzes, sondern um seine energiewirtschaftliche Verwendung zur Erzeugung von Elektrizität. Dieser Gehalt weist $§ 48$ KVBG klar als Regelung aus, die ausschließlich oder jedenfalls im Schwerpunkt dem Kompetenztitel „Energiewirtschaft" (Art. 74 Abs. 1 Nr. 11 GG) zuzuordnen ist. Denn darunter wird allgemein auch die Gewinnung von Primärenergiequellen und auch die Sicherung der Energieversorgung verstanden. ${ }^{11}$ Selbst wenn man $\$ 48 \mathrm{KVBG}$ im Sinne einer kompetenzrechtlichen Mehrfachzuordnung sowohl der Materie „Bergbau“ als auch der Materie „Energiewirtschaft" zuordnen wollte, würde dies, da beide Bestandteil des Art. 74 Abs. 1 Nr. 11 GG sind, nichts daran ändern, dass für $₫ 48$ KVBG die Anforderungen des Art. 72 Abs. 2 GG gelten. Der Umstand, dass der Gesetzentwurf der Bundesregierung (BT-Drs. 19/17342) die Gesetzgebungskompetenz für das Kohleverstromungsbeendigungsgesetz allein auf Art. 74 Abs. 1 Nr. 24 GG (Luftreinhaltung) stützen wollte, ${ }^{12}$ führt zu keinem anderen Ergebnis. Zunächst kommt es nicht auf die Entwurfsbegründung an, sondern auf die tatsächliche verfassungsrechtliche Lage. Selbst wenn das Kohleverstromungsbeendigungsgesetz insgesamt den Zweck der Verminderung von Treibhausgasen und somit des Klimaschutzes als anerkanntem Bestandteil des Kompetenztitels „Luftreinhaltung“ verfolgt, muss dies keine Auswirkung auf $₫ 48$ KVBG haben. Denn die Prüfung der Gesetzgebungskompetenz ist nicht auf ein Gesetz im Sinne der rechtstechnischen Einheit insgesamt zu beziehen, sondern muss in Zweifelsfällen für jede einzelne Regelung innerhalb des jeweiligen Gesetzes gesondert erfolgen. ${ }^{13}$ Festzuhalten bleibt deshalb, dass die gesetzgebungskompetenzrechtlich gesondert zu prüfende Vorschrift des $\$ 48$ KVBG auch dann ausschließlich oder jedenfalls ihrem Regelungsschwerpunkt nach auf den Kompetenztitel

7) BT-Drs. 19/20714 (neu), S. $168 \mathrm{f}$.

8) Allgemein verstanden im Sinne des Aufsuchens, Gewinnens und Aufbereitens von Bodenschätzen (Mineralien und Gesteine).

9) $\mathrm{Zu}$ der rechtlichen Grundlage des Braunkohletagebaus im Landesplanungsrecht einerseits und im Bundesberggesetz andererseits (und auch $\mathrm{zu}$ deren problematischem Verhältnis) s. nur BVerfG, Urt. v. 17.12.2013 - 1 BvR 3139, 3386/08, BVerfGE 134, 242, $244 \mathrm{ff}$. (Rdnr. 3 ff., $344 \mathrm{ff} ., 301 \mathrm{ff}$.)

10) V. 13.8.1980, BGBl. I.S. 1310; zuletzt geändert durch Art. 237 Elfte Zuständigkeitsanpassungsverordnung v. 19. 6.2020, BGBl. I. S. 1328.

11) S. nur Oeter, in: von Mangoldt/Klein/Starck, Grundgesetz, 7. Aufl. 2018, Art. 74 Rdnr. 89; Seiler, in: BeckOK Grundgesetz, Epping/Hillgruber, 45. Ed. (Stand: 15.11.2020), Art. 74 Rdnr. 36.

12) BT-Drs. 19/17342, S. 86: „Die Gesetzgebungskompetenz des Bundes für Artikel 1 dieses Gesetzes ergibt sich aus Artikel 74 Absatz 1 Nummer 24 Grundgesetz. Die Bestimmungen fallen in den Bereich der Luftreinhaltung. Ziel des Kohleausstiegsgesetzes ist die Reduzierung der Kohlendioxidemissionen, die durch die Erzeugung elektrischer Energie durch den Einsatz von Steinkohle erzeugt werden, um so das Klima als Teil der Umwelt zu schützen."

13) So hat etwa das Bundesverfassungsgericht etwa die Gesetzgebungskompetenz des Bundes spezifisch in Bezug auf $\int^{\circ} 43$ Abs. 1 des Weingesetzes geprüft, ohne nach der Kompetenzgrundlage für das Weingesetz insgesamt zu fragen: BVerfGE 136, 194, 241, Rdnr. 111. 
des Art. 74 Abs. 1 Nr. 11 GG (Energiewirtschaft/Bergbau) gestützt werden muss, wenn das gesamte Kohleverstromungsbeendigungsgesetz im Übrigen dem Kompetenztitel der Luftreinhaltung (Art. 74 Abs. 1 Nr. 24 GG) zuzuordnen wäre.

\section{Die aus Art. 72 Abs. 2 GG folgenden Maßstäbe}

Die bislang insbesondere in der Rechtsprechung des Bundesverfassungsgerichts erkennbaren Ansätze zur Konkretisierung der in Art. 72 Abs. 2 GG enthaltenen Maßstäbe sind nachfolgend als Grundlage für eine genauere Prüfung von \48 KVBG zusammenzufassen.

\subsection{Wahrung der Wirtschaftseinheit im gesamtstaatlichen Interesse}

Was zunächst das Kriterium der Wahrung der Wirtschaftseinheit angeht, so zielt dieses auf die Erhaltung der Funktionsfähigkeit des Wirtschaftsraums der Bundesrepublik Deutschland. ${ }^{14}$ Eine bundesgesetzliche Regelung ist nach der Rechtsprechung des Bundesverfassungsgerichts ,zur Wahrung der Wirtschaftseinheit erforderlich, wenn und soweit sie Voraussetzung für die Funktionsfähigkeit des Wirtschaftsraums der Bundesrepublik ist, wenn also unterschiedliche Landesregelungen oder das Untätigbleiben der Länder erhebliche Nachteile für die Gesamtwirtschaft mit sich brächten“. ${ }^{15}$ Es geht bei der Wahrung der Wirtschaftseinheit im Schwerpunkt darum, ,Schranken und Hindernisse für den wirtschaftlichen Verkehr im Bundesgebiet zu beseitigen“. Konkret hat das Bundesverfassungsgericht etwa mit Bezug auf eine Regelung, die den Deutschen Weinfonds betraf, darauf abgestellt, dass es um ,überregionale Maßnahmen“ und um Exportförderung gehe, die „,der deutschen Weinwirtschaft als Ganzer"16 zugutekomme. Eine bundesgesetzliche Bedarfsfeststellung für ein einzelnes Vorhaben nach der hier vorliegenden Art des $\$ 48$ KVBG war bislang nicht Gegenstand einer bundesverfassungsgerichtlichen Prüfung am Maßstab der Erforderlichkeit zur Wahrung der Wirtschaftseinheit im gesamtstaatlichen Interesse. ${ }^{17}$

\subsection{Doppelte Erforderlichkeitsprüfung (,wenn und soweit ... erforderlich")}

Das Merkmal der Erforderlichkeit einer bundesgesetzlichen Regelung zur Erreichung der in Art. 72 Abs. 2 GG genannten Zwecke - hier der Wirtschaftseinheit - wird durch den Bezug auf das ,gesamtstaatliche Interesse' in besonderer Weise geprägt. „Die Regelung durch Bundesgesetz muss danach nicht unerlässlich für die Rechts- oder Wirtschaftseinheit in dem normierten Bereich sein. Es genügt vielmehr, dass der Bundesgesetzgeber andernfalls nicht unerheblich problematische Entwicklungen in Bezug auf die Rechts- oder Wirtschaftseinheit erwarten darf “. ${ }^{18}$

Die Erforderlichkeitsprüfung hat nach der Rechtsprechung des Bundesverfassungsgerichts, die dabei überzeugend an den Wortlaut des Art. 72 Abs. 2 GG - ,wenn und soweit" - anknüpft, einen doppelten Bezugspunkt. Sie bezieht sich zunächst auf die Erforderlichkeit des Gesamtkonzepts eines Regelungswerks (grundsätzliche Regelungsbefugnis) und auf einer zweiten Stufe auf die Erforderlichkeit seiner einzelnen Elemente (zulässiges Ausmaß bundesgesetzlicher Regelungen), wobei sich die Frage der Teilbarkeit des Gesamtkonzepts stellen kann: „Hat der Bundesgesetzgeber auf einem ihm kompetentiell vom Grundgesetz gemäß Art. 74 Abs. 1 GG zugewiesenen Gebiet ein umfassendes Regelungskonzept entwickelt, das zum Schutz der Rechtsgüter des Art. 72 Abs. 2 GG nach Ziel und Wirkung erforderlich ist, können einzelne Teile dieses Regelungskonzepts mit dem zweiten Prüfungsschritt nur dann gemäß Art. 72 Abs. 2 GG (,soweit') als zu regelungsintensiv herausgenommen werden, wenn das Gesamtkonzept und damit die Wirkung des Gesetzes ohne sie nicht gefährdet wird". 19

\subsection{Verfassungsgerichtliche Kontrollintensität}

Nachdem im Jahr 1994 in Art. 72 Abs. 2 GG die Voraussetzung für die Wahrnehmung der konkurrierenden Gesetzgebungskompetenz durch den Bund von einer Bedürfnis- auf eine Erforderlichkeitsklausel umgestellt worden war, verschärfte das Bundesverfassungsgericht seinen zuvor stark zurückgenommenen Kontrollmaßstab deutlich. Die Einschätzungsprärogative des Gesetzgebers wird primär auf die tatsächlichen Entwicklungen bezogen, von denen die Erforderlichkeit bundesrechtlicher Regelungen abhängt, während auf dieser Tatsachengrundlage eine eingehende Kontrolle des in der gesetzlichen Regelung zum Ausdruck kommenden Konzepts vorgenommen wird. ${ }^{20}$ Die vorangegangene Rechtsprechung aufgreifend und zusammenfassend hat das Bundesverfassungsgericht dazu im Jahr 2015 ausgeführt:

„Ob die Voraussetzungen des Art. 72 Abs. 2 GG gegeben sind, hat das Bundesverfassungsgericht zu überprüfen. Insoweit besteht kein von verfassungsgerichtlicher Kontrolle freier gesetzgeberischer Beurteilungsspielraum ... Im Rahmen der danach eröffneten verfassungsgerichtlichen Kontrolle steht dem Gesetzgeber im Hinblick auf die allein zulässigen Zwecke einer bundesgesetzlichen Regelung und deren Erforderlichkeit im gesamtstaatlichen Interesse im Sinne von Art. 72 Abs. 2 $G G$ allerdings eine Einschätzungsprärogative zu ... Diese Prärogative des Bundesgesetzgebers bezieht sich insbesondere auf die Einschätzung und Bewertung tatsächlicher Entwicklungen, von denen die Erforderlichkeit bundesrechtlicher Regelung hinsichtlich der in Art. 72 Abs. 2 GG genannten Zwecke abhängt. Indessen reicht die Einschätzungsprärogative des Bundesgesetzgebers nicht so weit, dass die Beurteilung, ob die Voraussetzungen des Art. 72 Abs. 2 GG vorliegen, ganz in seine Hand gegeben wäre.

Dies gilt auch für die Beurteilung der ... Erforderlichkeit einer Regelung im Rahmen eines regulatorischen Gesamtkonzepts des Bundesgesetzgebers. Ungeachtet der Frage, inwieweit die Zugehörigkeit verschiedener Bestimmungen zu einem Gesamtkonzept überhaupt die Voraussetzungen des Art. 72 Abs. 2 GG auch für jene Regelungsteile begründen könnte, die diese Anforderungen für sich genommen nicht erfüllen ..., ist jedenfalls die Handhabung einer solchen potenziell kompetenzerweiternden Verknüpfungsmöglichkeit durch den Bundesgesetzgeber nicht von verfassungsrechtlicher Kontrolle freigestellt. Zwar verbleibt dem Gesetzgeber regelmäßig eine Prärogative für Konzept und Ausgestaltung von Gesetzen,

14) Broemel, in: v. Münch/Kunig, GG, 7. Aufl. 2021, Art. 72 Rdnr. 42, mit Verweis auf BVerfGE 106, 62, 146f.; 112, 226, 249; 122, 1, 22; Degenhart, in: Sachs, GG, 8. Aufl. 2018, Art. 72 Rdnr. 17.

15) BVerfGE 140, 65, 87, Rdnr. 49; dort auch das im Text folgende Zitat.

16) BVerfGE 136, 194, 242, Rdnr. 114.

17) Einen knappen Überblick über die bislang entschiedenen Regelungen, die vom Recht der Altenpflege über Agrarmarktbeihilfen und Mindestabsatzregelung für die Gewerbesteuer zur Vermeidung von Steueroasen bis hin zu Regelungen zur Filmförderung und zu Erbschafts- und Schenkungssteuern reichen, bei Broemel, in: v. Münch/Kunig, GG, 7. Aufl. 2021, Art. 72 Rdnr. 43, mit Nachw. der Rechtsprechung.

18) BVerfGE 140, 65, 88, Rdnr. 49

19) BVerfGE 113, 167, 197; nachfolgend ähnlich BVerfGE 140, 65, 95, Rdnr. 66. S auch Broemel, in: v. Münch/Kunig, GG, 7. Aufl. 2021, Art. 72 Rdnr. 35 mit Fn. 203.

20) Zusammenfassend mit neueren Nachweisen der Rechtsprechung Broemel, in: v. Münch/Kunig, GG, 7. Aufl. 2021, Art. 72 Rdnr. 35. 
was einschließt, eine politische Verbindung zwischen eigenständigen Instrumenten der Fürsorge herzustellen. Ob einzelne Bestandteile des politischen Gesamtkonzepts im Sinne des Art. 72 Abs. 2 GG erforderlich sind, weil sie nicht entfallen könnten, ohne das Gesamtkonzept zu gefährden, ist jedoch eine Frage des Verfassungsrechts, die gerichtlicher Kontrolle nicht vollständig entzogen ist ...."21

\section{Erforderlichkeit der Bedarfsfeststellung für Garzweiler II zur Wahrung der Wirtschaftseinheit im gesamtstaatlichen Interesse?}

\subsection{Regelungsgegenstand und planungsrechtssystematische Einordnung}

Alleiniger Regelungsgegenstand von $₫ 48 \mathrm{KVBG}$ ist der Braunkohletagebau Garzweiler II, wie er durch Entscheidung der Landesregierung Nordrhein-Westfalen auf der Grundlage des Landesplanungsgesetzes (\$29 Abs. 2) durch Leitentscheidung vom 5. Juli 2016 räumlich eingegrenzt wurde. Dieser räumlich und gegenständlich begrenzte Anwendungsbereich des $\$ 48$ KVBG qualifiziert diese Norm als Maßnahmegesetz, das mit seiner bedarfsfeststellenden Wirkung planungsrechtssystematisch in der Nähe gesetzlicher Bedarfsplanungen angesiedelt $\mathrm{zu}$ sein scheint, wie sie in Form des Bundesfernstraßenausbaugesetzes, ${ }^{22}$ des Bundesschienenwegeausbaugesetzes ${ }^{23}$ und des Bundesbedarfsplangesetzes ${ }^{24}$ für den Ausbau der Elektrizitäts-Übertragungsnetze bekannt sind. Allerdings sind diese drei Erscheinungsformen gesetzlicher Bedarfsplanung auf das gesamte Territorium der Bundesrepublik Deutschland bezogen, weshalb - soweit ersichtlich - bislang keinerlei Zweifel an der Vereinbarkeit dieser Bedarfsplanungsgesetze mit Art. 72 Abs. 2 GG aufgekommen sind. ${ }^{25}$ Sie nehmen gesamtstaatlich erforderliche Netzinfrastrukturen in den Blick und entscheiden prognostisch über den Ausbaubedarf auf der Grundlage einer Analyse des Bestandes, der prognostizierten Entwicklung und der Angemessenheit des Mitteleinsatzes. ${ }^{26}$

Nichts von alledem zeichnet die Bedarfsfeststellung in $\$ 48 \mathrm{KVBG}$ aus. Sie ist allein bezogen auf einen einzelnen Tagebau und nimmt weder nach ihrem Regelungsgegenstand noch nach ihrer Begründung oder den erkennbaren Grundlagen Bezug auf eine gesamtstaatliche Perspektive. ${ }^{27}$ Die Erforderlichkeit der Bedarfsfeststellung für Garzweiler II zur Wahrung der Wirtschaftseinheit im gesamtstaatlichen Interesse erscheint vor diesem Hintergrund besonders begründungsbedürftig.

\subsection{Erforderlichkeit zur gesamtstaatlichen Sicherung der Energieversorgung?}

Der einzige Grund für eine Erforderlichkeit des $₫ 48$ KVBG zur Wahrung der Wirtschaftseinheit im gesamtstaatlichen Interesse könnte sich daraus ergeben, dass diese Vorschrift allein oder als Teil eines gesetzlichen Gesamtkonzepts dazu dient, die gesamtstaatliche Sicherheit der Energieversorgung sicherzustellen.

\subsubsection{Kein bundesgesetzliches Regelungskonzept zum Braunkohletagebau}

Ein solcher Beitrag des $\$ 48$ KVBG zur gesamtstaatlichen Energieversorgungsicherheit würde allerdings voraussetzen, dass das KVBG - alleine oder auch im Verbund mit anderen bundesgesetzlichen Regelungen - ein Konzept zur Gewährleistung der Energieversorgungssicherheit enthält, welches mögliche Braunkohleabbauflächen bzw. -standorte aus gesamtstaatlicher Perspektive in den Blick nimmt und unter Berücksichtigung raumplanerischer, energiewirtschaftlicher und weiterer Kriterien einer Bedarfsplanung unterwirft. Ein solches Konzept ist aber weder im KVBG noch in anderen Bundesgesetzen erkennbar. Le- diglich der in Anlage 2 zum KVBG enthaltenen Liste der Stilllegungszeitpunkte von Braunkohleanlagen im gesamten Bundesgebiet lässt sich ein Konzept aus gesamtstaatlicher Perspektive entnehmen. Dieses Konzept ist aber nicht auf Braunkohletagebaue bezogen und verhält sich nicht zu der Frage, aus welchem Braunkohletagebau die mit Stilllegungszeitpunkt aufgelisteten Braunkohlekraftwerksblöcke versorgt werden. Dabei zeigt ein Blick auf die tatsächliche und rechtliche Situation der Braunkohletagebauflächen in den verschiedenen Braunkohlerevieren, dass durchaus Anlass bestanden hätte, über Garzweiler II hinaus eine Bedarfsplanung für Tagebaue im gesamtstaatlichen Interesse an einer sicheren Energieversorgung unter Berücksichtigung des Klimaschutzes und anderer öffentlicher Belange zu betreiben. Wie die Kommission „Wachstum, Strukturwandel und Beschäftigung" in ihrem Bericht dargelegt hat, sind die heute betriebenen und projektierten Braunkohletagebaue ,das Resultat eines komplexen genehmigungsrechtlichen Gefüges, bestehend aus landespolitischen Leitentscheidungen, Braunkohlenplänen, Rahmen sowie Hauptbetriebsplänen. Sie beruhen auf einer zeitintensiven Abstimmung zwischen verschiedenen Interessen und der Abwägung der Auswirkungen eines Tagebaus auf Grundlage der bestehenden rechtlichen Rahmenbedingungen"“. ${ }^{28}$ Planungen und fachrechtliche Zulassungen, für die die Bedarfsfeststellung nach $\ 48 \mathrm{KVBG}$ Bindungswirkung entfalten soll ( $\$ 48$ Abs. 2 Satz $1 \mathrm{KVBG})$, stehen nicht nur für Garzweiler II aus, sondern für eine Reihe weiterer Tagebaue. ${ }^{29}$ Die bundesgesetzlich mit Bindungswirkung für nachfolgende Planungs- und Zulassungsentscheidungen ausgestattete Feststellung, dass Garzweiler II energiewirtschaftlich notwendig ist, könnte zur gesamtstaatlichen Sicherung der Energieversorgung im Sinne des Art. 72 Abs. 2 GG nur dann erforderlich sein, wenn sie eingebettet wäre in eine Bedarfsprüfung anderer Braunkohlestandorte. Denn die Feststellung eines Bedarfs ohne die Berücksichtigung von Alternativen ist ein Widerspruch in sich. Da der Bundesgesetzgeber seinen Blick nicht auf die anderen Braunkohletagebaue in Deutschland gerichtet und über deren energiewirtschaftliche Erforderlichkeit keine Aussage getroffen hat, lässt er genau die gesamtstaatliche Perspektive

21) BVerfGE 140, 65, $94 \mathrm{f} ., \mathrm{Rdnr} .65 \mathrm{f}$.

22) Fernstraßenausbaugesetz in der Fassung der Bekanntmachung $v$. 20.1.2005, BGBl. I S. 201; zuletzt geändert durch Art. 1 des Gesetzes v. 23.12.2016, BGBl. I S. 3354

23) Bundesschienenwegeausbaugesetz v. 15.11.1993, BGBl. I S. 1874; zuletzt geändert durch Art. 1 des Gesetzes v. 23.12.2016, BGBl. I S. 3221.

24) Bundesbedarfsplangesetz v. 23.7.2013, BGB1. I S. 2543; 2014 I S. 148, 271; zuletzt geändert durch Art. 3 des Gesetzes v. 13. 5. 2019, BGBl. I S. 706.

25) Da das Fernstraßenausbaugesetz auf Art. 74 Abs. 1 Nr. 22 GG und das Bundesbedarfsplangesetz auf Art. 74 Abs. 1 Nr. 11 GG (Energiewirtschaft) beruht, gilt für diese beiden Bedarfsplanungsgesetze Art. 72 Abs. 2 GG, während dies bei dem Bundesschienenwegeausbaugesetz (Art. 73 Abs. 1 Nr. 6a GG) nicht der Fall ist.

26) Zur Funktion und den Charakteristika einer Bedarfsplanung Köck, ZUR 2016, S. $579 \mathrm{ff}$.

27) Das gilt auch für die in der Begründung zu $₫ 48$ KVBG in Bezug genommenen Ergebnisse der Kommission „Wachstum, Strukturwandel und Beschäftigung", in denen sich keine Aussagen zum energiewirtschaftlichen Bedarf einzelner oder mehrerer Braunkohletagebaue in gesamtstaatlicher Perspektive finden.

28) Kommission „Wachstum, Strukturwandel und Beschäftigung“, Abschlussbericht, Januar 2019, S. 41.

29) S. dazu die Angaben in BET, Gutachten: Ermittlung von Folgekosten des Braunkohletagebaus bei einem gegenüber aktuellen Braunkohle- bzw. Revierplänen veränderten Abbau und Bestimmung der entsprechenden Rückstellungen, Gutachten im Auftrag des BMWi, Aachen, 14.12.2020, u.a.S. 58 (Tagebau Nochten/Lausitz), S. 59f. (Tagebau Sonderfeld Mühlrose), S. 95 (Profen/Mitteldeutsches Revier). 
vermissen, die Art. 72 Abs. 2 GG von ihm zwingend verlangt. Für den isolierten Blick nur auf Garzweiler II ist der Landesgesetzgeber offensichtlich der sach- und problemnähere föderale Akteur, dessen Regelungsbefugnis Art. 72 Abs. 2 GG gegen nicht erforderliche Übergriffe des Bundes abschirmen will.

4.2.2 \$48 ohne Bezug zu bundesgesetzlichen Regelungen der Energieversorgungssicherheit

Ein Beitrag des $\ 48$ KVBG zur Gewährleistung gesamtstaatlicher Energieversorgungssicherheit in Deutschland ist auch deshalb nicht ersichtlich, weil diese Vorschrift in keinerlei Beziehung zu den Instrumenten des Energiewirtschaftsgesetzes und des Kohleverstromungsbeendigungsgesetzes steht, die die Versorgungssicherheit gewährleisten sollen.

Das Recht der Energieversorgungssicherheit sieht sich bekanntlich mit den Grundstrukturen eines liberalisierten Energiemarktes konfrontiert, so dass die staatliche Gewährleistung der Energieversorgungssicherheit nur mit Hilfe der Marktakteure - insbesondere der Erzeugungsunternehmen - funktionieren kann. Auf der Grundlage unionsrechtlicher Vorgaben beruht das Recht der Energieversorgungssicherheit deshalb in erster Linie auf einer systematischen Beobachtung der Energieversorgungssituation, die in $\ 51$ EnWG (Monitoring der Versorgungssicherheit) dem Bundesministerium für Wirtschaft und Energie zugewiesen und mit den erforderlichen Befugnissen zur Erhebung von Informationen bei den Energieversorgungsunternehmen (bzw. mit Meldepflichten) ausgestattet ist. ${ }^{30}$ Seit 2016 ist dieses Monitoring der Versorgungssicherheit ergänzt durch die Möglichkeit der Regulierungsbehörde, ein eigenständiges Monitoring des Beitrags von Lastmanagement zur Versorgungssicherheit einzuführen (\$51a EnWG) und zu diesem Zweck Informationen bei Großverbrauchern zu erheben. Für den Fall, dass sich als Ergebnis dieser Beobachtungen drohende Versorgungslücken oder -engpässe zeigen, hält das Energierecht im Vertrauen auf die Kräfte des Marktes allerdings nur begrenzte staatliche (Zwangs-)Instrumente bereit, zu denen die Verpflichtung zur Vorratshaltung von Brennstoffen ( $(50$ EnWG) und die Möglichkeit gehören, ein Ausschreibungsverfahren für neue Erzeugungskapazitäten (bzw. Energieeffizienz- und Nachfragesteuerungsmaßnahmen) einzuführen ( $\$ 53$ EnWG). Die Elektrizitätsversorgungssicherheit sieht das Energiewirtschaftsgesetz einerseits durch das „Bilanzkreis- und Ausgleichsenergiesystem“ gewährleistet ( $\$ 1$ a Abs. 2 EnWG), indem es die Bilanztreue der Bilanzkreisverantwortlichen und die ordnungsgemäße Bewirtschaftung der Bilanzkreise stärkt ( $\$ 13$ Abs. 5 S. 2 EnWG). Andererseits sieht das Gesetz einer Reihe von speziellen Markteingriffen der Übertragungsnetzbetreiber zur Gewährleistung der Versorgungssicherheit vor. Diese reichen von der Steuerung des Einspeiseverhaltens von Kraftwerken diesseits und jenseits eines Netzengpasses (Redispatch, $\$ 13$ a EnWG) über das Vorhalten einer Netzreserve ( $\$ 13 \mathrm{~d}$ EnWG), die insbesondere aus ,eigentlich“" stillzulegenden, wegen ihrer Systemrelevanz aber betriebsbereit zu haltenden Anlagen besteht ( $\$ 13 \mathrm{~b}$ EnWG), ${ }^{31}$ bis hin zu einem durch Ausschreibungsverfahren zu bildenden ,Kapazitätspuffer" für kurzfristig auftretende, außergewöhnliche Situationen (Kapazitätsreserve, $\$ 13 \mathrm{e}$ EnWG). ${ }^{32}$ Speziell für stillgelegte Braunkohlekraftwerke sieht das Gesetz schließlich eine zeitlich begrenzte Sicherheitsbereitschaft vor, die für die bereits zwischen 2016 und 2019 stillgelegten Braunkohlekraftwerke in $\$ 13 \mathrm{~g}$ EnWG normiert ist und für einige der ab 2020 stillzulegenden Kraftwerksblöcke in $\int S 40 \mathrm{ff}$. KVBG näher ausgestaltet ist. Darüber hinaus ergänzt $\$ 34$ KVBG die allgemeinen Beobachtungsinstrumente des EnWG durch ein spezielles Monitoring-Instrument, das sich speziell auf die Auswirkungen der Reduzierung der Stein- und Braunkohleverstromung bezieht. Aus dieser knappen Übersicht wird erkennbar, dass das Energiewirt- schaftsrecht auf der Grundlage eines marktbasierten Elektrizitätsversorgungssystems ein begrenztes Instrumentarium zur Gewährleistung der Versorgungssicherheit kennt. An keiner Stelle in diesem System ist aber ersichtlich, dass der Gesetzgeber Versorgungssicherheit dadurch gewährleisten will, dass er Stromproduzenten den Zugang zu bestimmten primären Energieträgern an einem bestimmten Ort zusichert oder garantiert. Adressaten des Energieversorgungssicherheitsrechts sind auf der Erzeugerseite die Betreiber von Kraftwerken, die sich im Rahmen des allgemeinen Rechts nach Marktgesetzen ihre Kraftwerkstandorte zu suchen und ihre Brennstoffe zu beschaffen haben. Daran ändern auch die nach $\$ 4$ Abs. 1 KVBG i. V.m. Anlage 2 gestaffelten Stilllegungszeitpunkte für Braunkohlekraftwerke nichts, weil diese nur den spätesten Zeitpunkt der Stilllegung bestimmen und das erläuterte Konzept der Elektrizitätsversorgungssicherheit weder ergänzen noch modifizieren. Es bleibt dabei, dass der Bundesgesetzgeber den Zugriff von Kraftwerksbetreibern auf die Primärenergiequellen unter dem Gesichtspunkt der Versorgungssicherheit nicht durch eine nationales Planungs-, Bewirtschaftungs- oder Gewährleistungsregime geregelt hat. Insoweit stellen bundesweit die einzige Ausnahme die beiden Braunkohlekraftwerke in Neurath und Niederaußem dar, zu deren ,planungssicherer Versorgung“ der Tagebau Garzweiler II ,als einziger Tagebau" 33 verblieb und offenbar deshalb durch Bundesgesetz gesichert werden sollte.

4.2.3 Entscheidung über Braunkohleabbau fällt in die Zuständigkeit der Länder

Stattdessen ist es nach der verfassungsrechtlichen Verteilung der Gesetzgebungs- und Verwaltungszuständigkeiten zwischen Bund und Ländern und auf der Grundlage der Regelungen im BBergG, im Raumordnungsgesetz des Bundes und im Landesplanungsgesetz des Landes Nordrhein-Westfalen allein Sache der zuständigen Organe des Landes Nordrhein-Westfalen, darüber zu entscheiden, ob der Tagebau Garzweiler II energiewirtschaftlich notwendig ist. Dies gilt jedenfalls so lange, wie der Bundesgesetzgeber keine nationale Braunkohletagebaubedarfsplanung installiert hat. Wie das Bundesverfassungsgericht in seiner Garzweiler-Entscheidung aus dem Jahr 2013 ausdrücklich aus verfassungsrechtlicher Perspektive bestätigt hat, werden die „Leitentscheidungen für das langfristige Konzept des Braunkohlenabbaus im Rheinischen Braunkohlengebiet auf der Ebene der Landesregierung des Landes NordrheinWestfalen getroffen“, da ,es um eine zentrale Frage der Energiepolitik des Landes geht". ${ }^{34}$ Gleichzeitig hat das Gericht festgestellt, dass die Kompetenz zur Entscheidung der Grundsatzfrage für den Abbau von Braunkohle im Rheinischen Braunkohlenrevier oder in einem anderen Revier nicht durch Gesetz dem Bund übertragen worden ist. ${ }^{35}$ Eine solche Übertragung hat auch $\$ 48 \mathrm{KVBG}$ weder intendiert noch bewirkt. Vielmehr wurde eine punktuelle Standortentscheidung im Überschneidungsbereich von Raumordnung und Landesplanung einerseits und Bergrecht andererseits der nordrhein-westfälischen Exekutive aus der Hand

30) S. etwa den Monitoring-Bericht des Bundesministeriums für Wirtschaft und Energie nach $\int 51$ EnWG zur Versorgungssicherheit im Bereich der leitungsgebundenen Versorgung mit Elektrizität, Stand: Juli 2016; Bundesministerium für Wirtschaft und Energie, Versorgungssicherheit bei Erdgas - Monitoringbericht nach $\int 51$ EnWG, Stand: Juli 2017.

31) Dazu Steffens, VerwArch 2014, 313, $335 \mathrm{ff}$.

32) S. die Analyse dieser Instrumente bei Franke, in: FS SchmidtPreuß, 2018, S. 809, 811 ff.; s. auch den Überblick von Ruttloff/ Lukosek, GewArch 2019, $12 \mathrm{ff}$.

33) So die Begründung zu $₫ 48$ KVBG in dem Bericht des Ausschusses, BT-Drs. 19/20714 (neu), S. 169.

34) BVerfGE 134, 242, 346, Rdnr. 306

35) BVerfGE 134, 242, 347, Rdnr. 306. 
genommen. Ob darin ein Misstrauen des Bundesgesetzgebers gegenüber aktuellen oder zukünftigen Entscheidungen der zuständigen nordrhein-westfälischen Stellen beim Vollzug des maßgeblichen Bundesrechts zum Ausdruck kommt, lässt sich anhand der Materialien nicht belegen. Aber eine Rechtfertigung dieser gesetzgeberischen Intervention als erforderlich zur gesamtstaatlichen Sicherung der Energieversorgung lässt sich ebenfalls nicht feststellen.

\section{Ergebnis: Nichtigkeit des $₫ 48 \mathrm{KVBG}$}

Bei $₫ 48$ des Kohleverstromungsbeendigungsgesetzes, der die energiepolitische und energiewirtschaftliche Notwendigkeit und den vordringlichen Bedarf zur Gewährleistung einer sicheren und zuverlässigen Energieversorgung für den Tagebau Garzweiler II feststellt, handelt es sich um eine Regelung, die nur auf die Gesetzgebungskompetenz des Bundes aus Art. 74 Abs. 1 Nr. 11 GG (Energiewirtschaft/Bergbau) gestützt werden kann. $₫ 48$ KVBG unterliegt deshalb den Anforderungen des Art. 72 Abs. 2 GG, müsste also zur Wahrung der Wirtschaftseinheit im gesamtstaatlichen Interesse erforderlich sein. Diese Anforderung unterliegt der verfassungsgerichtlichen Kontrolle. Als gesamtstaatliches Interesse zur Rechtfertigung der bundesgesetzlichen Regelung in $\$ 48 \mathrm{KVBG}$ am Maßstab des Art. 72 Abs. 2 GG kommt allein die nationale Energieversorgungssicherheit in Betracht. Zur Gewährleistung der nationalen Energieversorgungssicherheit ist die gesetzliche Bedarfsfeststellung in $\$ 48 \mathrm{KVBG}$ aber nicht erforderlich, weil sie nicht auf einer Prüfung und Bewertung der energiewirtschaftlichen Notwendigkeit aller relevanten Braunkohletagebaue in Deutschland beruht (Fehlen einer nati- onalen Braunkohletagebaubedarfsplanung) und weil sie keinerlei Bezug zu dem gesetzlichen Konzept der Gewährleistung von Energieversorgungssicherheit aufweist. Stattdessen erweist sich $\$ 48 \mathrm{KVBG}$ als punktueller Übergriff des Bundesgesetzgebers in die exekutiven Planungs- und Vollzugskompetenzen der zuständigen Organe des Landes Nordrhein-Westfalen, der der ,planungssicheren Versorgung der Braunkohlekraftwerke in Neurath und Niederaußem" (Gesetzesbegründung) dient, nicht aber der Wahrung der Wirtschaftseinheit im gesamtstaatlichen Interesse im Sinne des $\$ 72$ Abs. 2 GG. $\$ 48$ KVBG ist deshalb mangels Gesetzgebungskompetenz des Bundes nichtig.

Open Access. Dieser Artikel wird unter der Creative Commons Namensnennung 4.0 International Lizenz veröffentlicht, welche die Nutzung, Vervielfältigung, Bearbeitung, Verbreitung und Wiedergabe in jeglichem Medium und Format erlaubt, sofern Sie den/die ursprünglichen Autor(en) und die Quelle ordnungsgemäß nennen, einen Link zur Creative Commons Lizenz beifügen und angeben, ob Änderungen vorgenommen wurden.

Die in diesem Artikel enthaltenen Bilder und sonstiges Drittmaterial unterliegen ebenfalls der genannten Creative Commons Lizenz, sofern sich aus der Abbildungslegende nichts anderes ergibt. Sofern das betreffende Material nicht unter der genannten Creative Commons Lizenz steht und die betreffende Handlung nicht nach gesetzlichen Vorschriften erlaubt ist, ist für die oben aufgeführten Weiterverwendungen des Materials die Einwilligung des jeweiligen Rechteinhabers einzuholen

Weitere Details zur Lizenz entnehmen Sie bitte der Lizenzinformation auf http://creativecommons.org/licenses/by/4.0/deed.de.

Open Access funding enabled and organized by Projekt DEAL.

\title{
Öffentlichkeitsbeteiligung nach Art. 6 und Zugang zu Gerichten nach Art. 9 Abs. 2 der Aarhus-Konvention
}

\author{
Zur Umsetzung im Unions- und im deutschen Recht*
}

Thomas Bunge

\begin{abstract}
(C) Springer-Verlag GmbH Deutschland, ein Teil von Springer Nature 2021
Ein neues Urteil des Europäischen Gerichtshofs (C-826/18) macht erneut deutlich, dass innerstaatliche Vorschriften über die materielle Präklusion verspäteter Einwendungen unzulässig sind, wenn sie Rechtsbehelfe aufgrund des Art. 9 Abs. 2 der Aarhus-Konvention betreffen, nicht aber, wenn sie für Rechtsbehelfe gemäß Art. 9 Abs. 3 der Konvention gelten. Der folgende Beitrag befasst sich deshalb mit der Frage, wie weit der Anwendungsbereich des Art. 9 Abs. 2 reicht. Diese Vorschrift gewährleistet den Zugang zu Gerichten in allen Fällen, in denen sich die Öffentlichkeit nach Art. 6 der Konvention an behördlichen Verfahren zur Zulassung von bestimmten Tätigkeiten beteiligen kann. Dazu gehören nicht allein die in Anhang I der Konvention aufgezählten Aktivitäten, sondernnach der teilweise unterschätzten Regelung des Art. 6 Abs. 1 Buchst. $b$ - auch andere Tätigkeiten, die sich erheblich auf die Um-
\end{abstract}

Dr. Thomas Bunge,

Direktor und Prof. beim Umweltbundesamt a. D.,

Honorarprofessor für Umwelt- und Planungsrecht

an der Technischen Universität Berlin a.D.,

Berlin, Deutschland welt auswirken können. Das deutsche Recht entspricht diesen Vorgaben weitgehend, aber nicht vollständig. Nicht in allen Fällen besteht ein Recht der Öffentlichkeit zur Partizipation an den relevanten Zulassungsverfahren; soweit es existiert, enthalten einige der einschlägigen Gesetze Bestimmungen über die materielle Präklusion. Andererseits gibt es keine innerstaatlichen Vorschriften, die über die zwingenden Anforderungen des Art. 9 Abs. 2 hinausgehen. Die materielle Präklusion ließe sich deswegen nicht ohne Anderung der Konvention und des Unionsrechts wieder einführen.

\section{Einleitung}

Art. 9 Abs. 2 der Aarhus-Konvention (AK) verlangt von den Vertragsparteien, Mitgliedern der betroffenen Öffentlichkeit unter den dort genannten Voraussetzungen Zugang zu Gerichten zu gewähren, „um die materiell-rechtliche und verfahrensrechtliche Rechtmäßigkeit von Entscheidungen, Handlungen oder Unterlassungen anzufechten,

^) Herrn Professor Dr. Peter-Christoph Storm zum 85. Geburtstag. 